\title{
Exploring the core microbial commonalities of alcoholics and CRC patients'
}

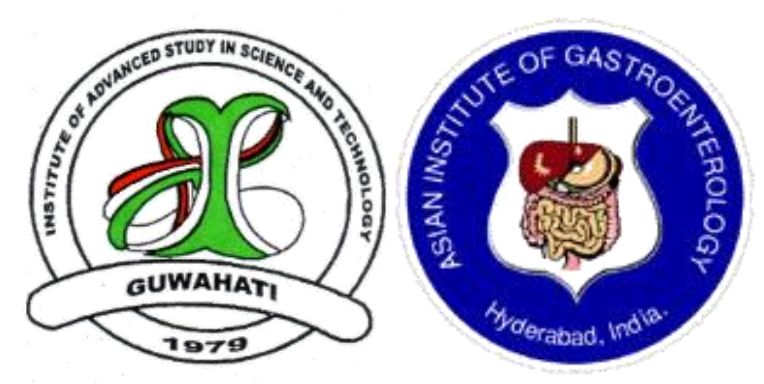

Priyanka Sarkar ${ }^{1,2 *}$, Mohan Chandra Kalita ${ }^{3}$, Rupjyoti Talukdar'1, Mojibur R. Khan²* ${ }^{1}$ DBT-Wellcome (Indian Alliance) Lab, Asian Healthcare Foundation, Asian Institute of Gastroenterology

${ }^{2}$ Institute of Advanced Study in Science and Technology (IASST)

${ }^{3}$ Dept. of Biotechnology, Gauhati University

*mojibur.khan@gmail.com and priyanka.sarkar14@gmail.com 


\section{ScienceNews}

\section{Origins of alcohol consumption traced to ape ancestor}

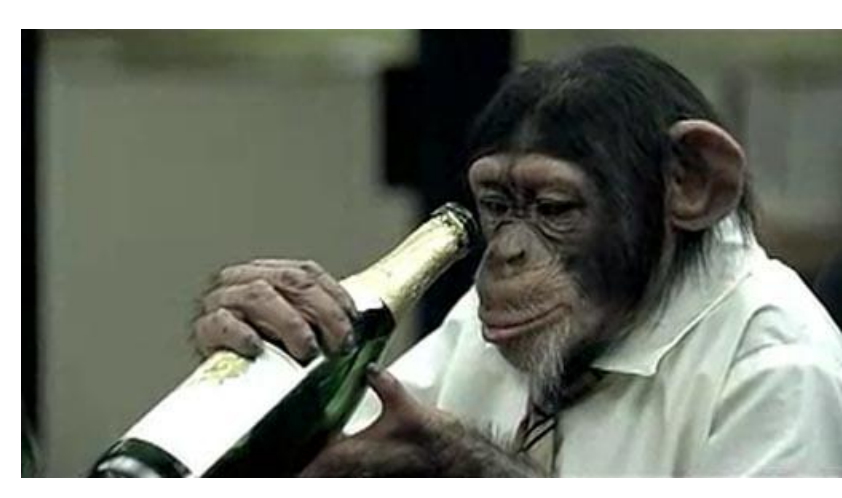

Eating fermented fruit off
BYERIN WAYMAN 9:22AM, FEBRUARY 18, 2013
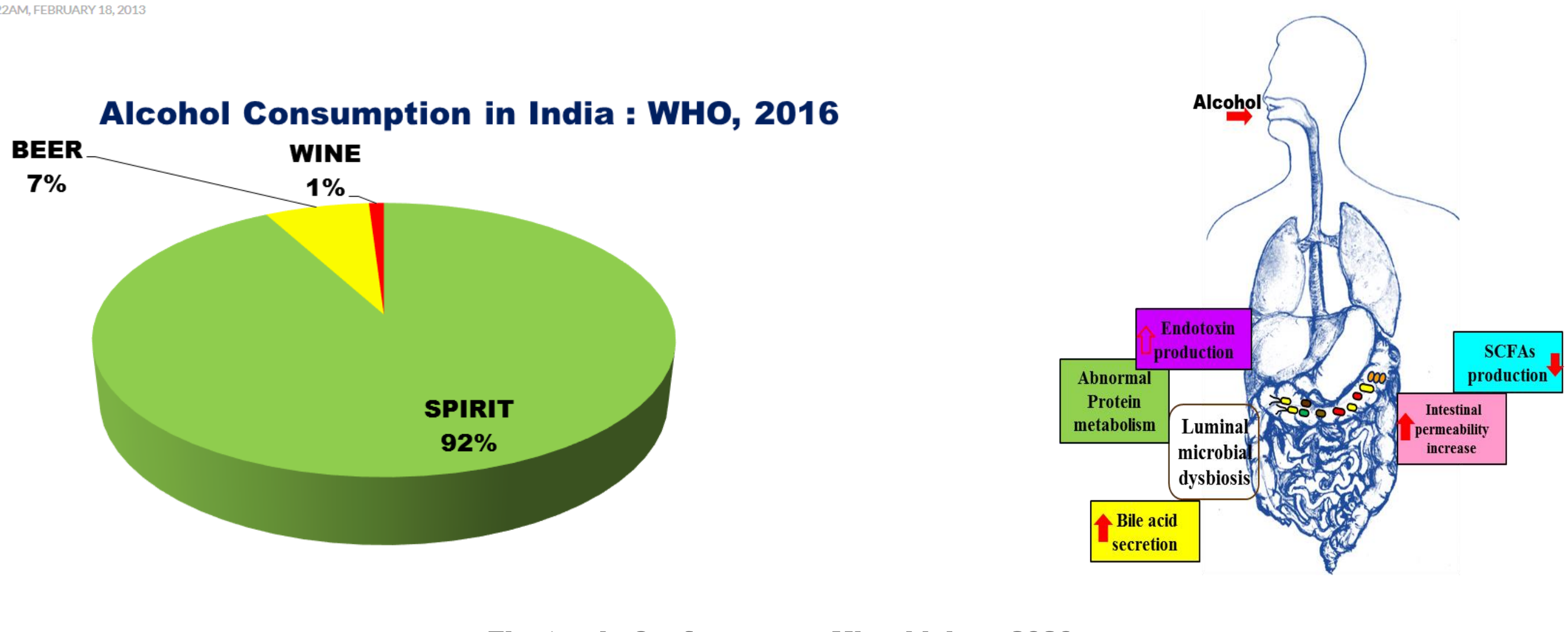


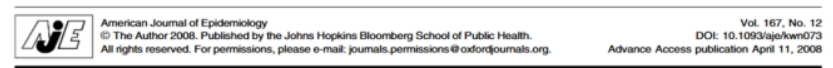

\section{Meta-Analysis}

Alcohol Drinking and Colorectal Cancer in Japanese: A Pooled Analysis of
Results from Five Cohort Studies

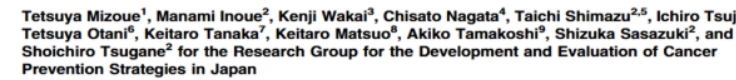

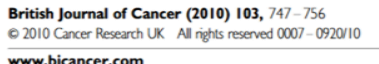

จำ

Alcohol intake and risk of colorectal cancer: Results from the UK Dietary Cohort Consortium

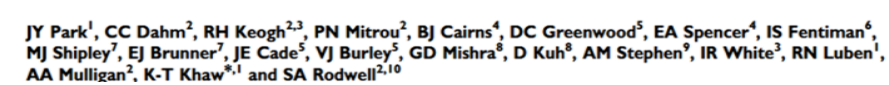

review

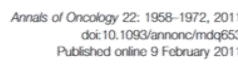

Alcohol drinking and the risk of colorectal cancer death: a meta-analysis

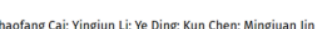

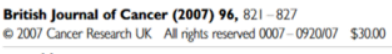

Cigarettes and alcohol in relation to colorectal cancer: the Singapore Chinese Health Study

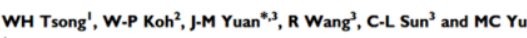

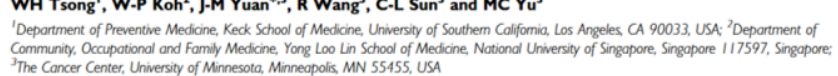

\section{BJC}

FULL PAPER

\section{Alcohol consumption and site-specific} cancer risk: a comprehensive dose-response meta-analysis

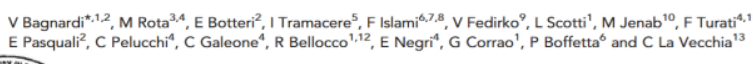

Alcohol drinking and colorectal cancer risk: an overall and dose-response meta-analysis of published studies

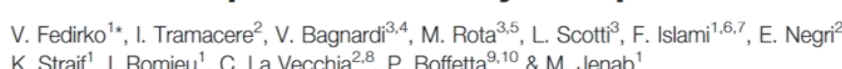

\section{EACP}

Annals of Internal Medicine'

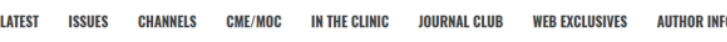

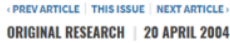

Alcohol Intake and Colorectal Cancer: A Pooled Analysis of 8 Cohort Studies

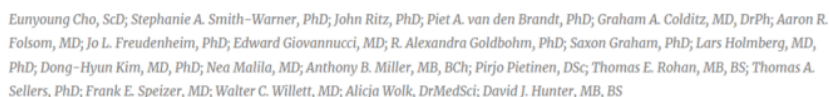

nature

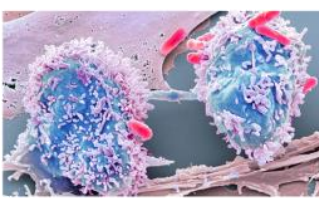

1. Altmeric 42 citations 202

The gut microbiota, bacterial metabolites and colorectal cancer

perat Louis, Gergina L L Hold a Harry. Filint

\section{SCIENTIFIC REP RTS}

OPEN Distinct gut microbiome patterns associate with consensus molecular subtypes of colorectal cancer

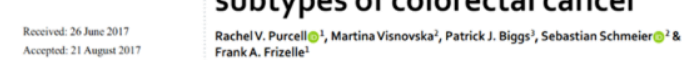

\section{HHS Public Access}

Author manuscript hor manuscript; available in PMC 2017 September 08. Published in final edited form a:

Gut Microbiota, Inflammation, and Colorectal Cancer Caitlin A. Brennan ${ }^{1}$ and Wendy S. Garrett $1,2,3,4$

\section{M. $९$ World Journal of Y Gastroenterology}

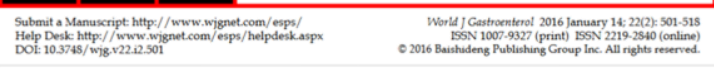
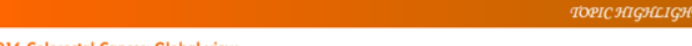

Gut microbiota imbalance and colorectal cancer

Johan Gogniere, Jenníer Raisch, Julie Veziant, Nicolas Bamich, Richard Bonnet, Emmanuel Buc,
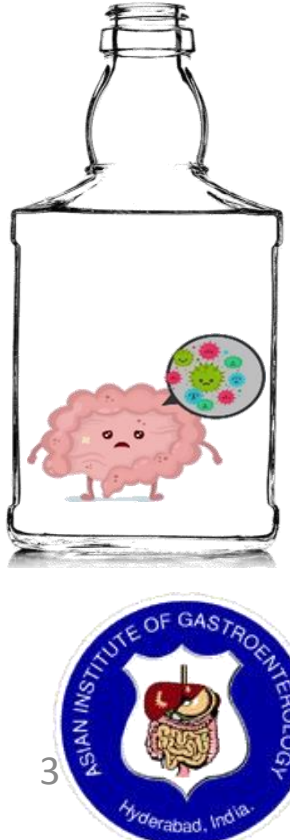


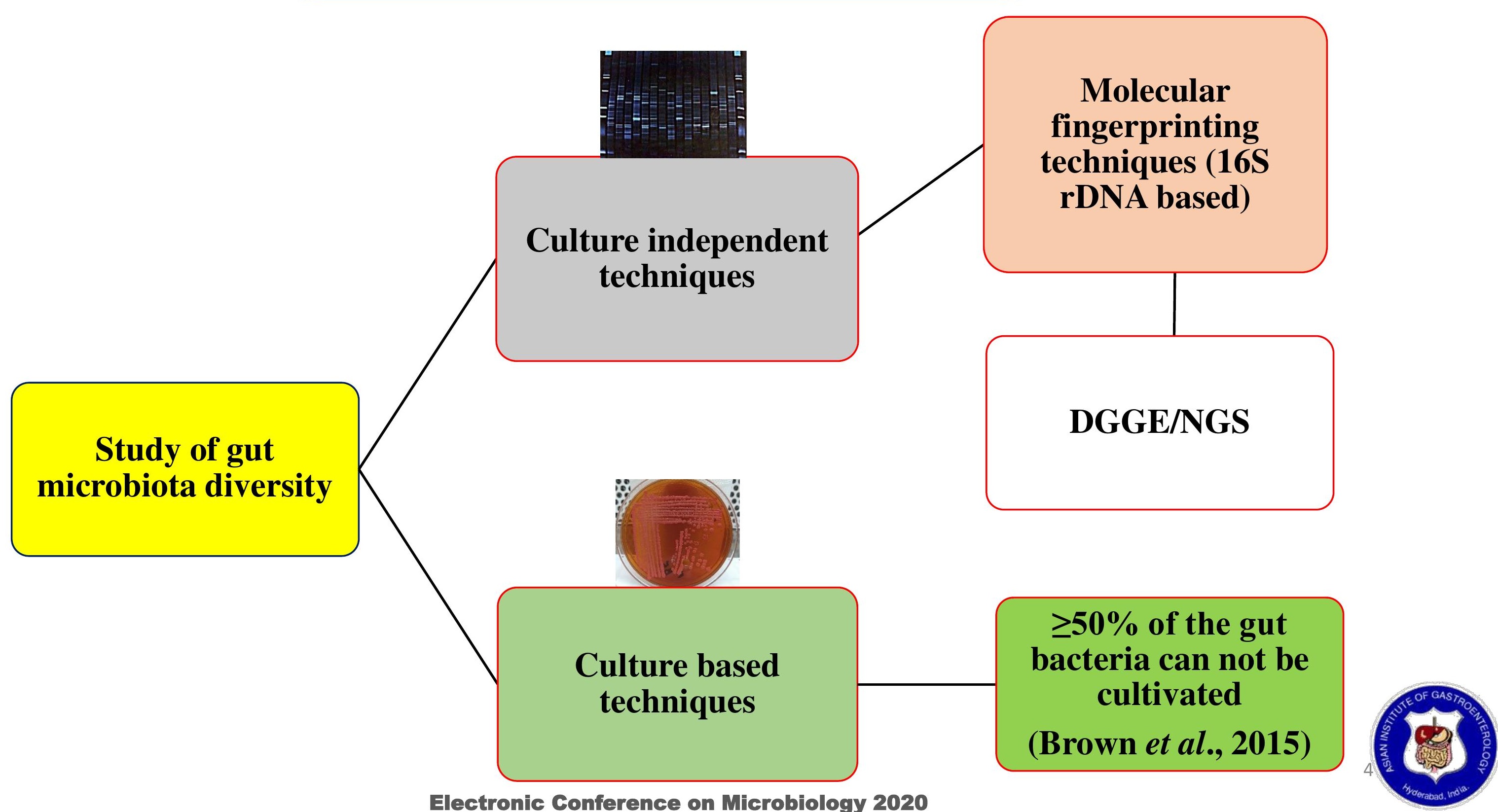




\section{Methodology used for the study}

\section{MG-RAST metagenomics analysis server

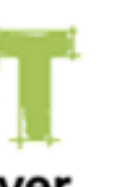

Publimed

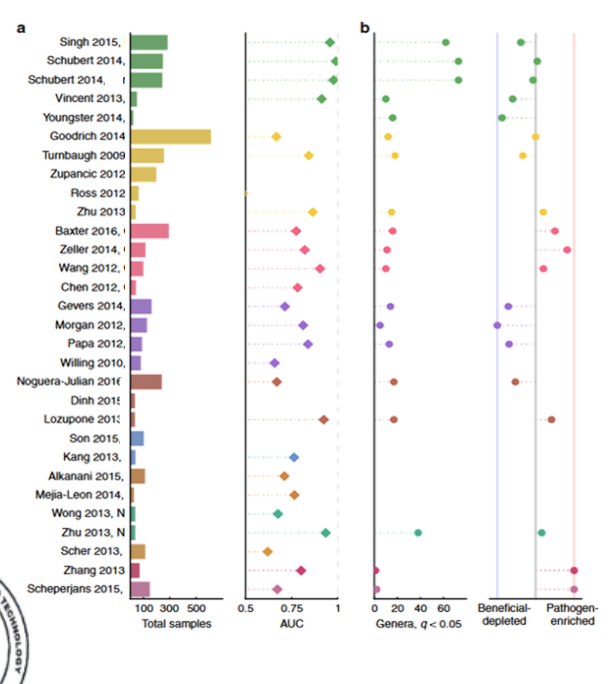

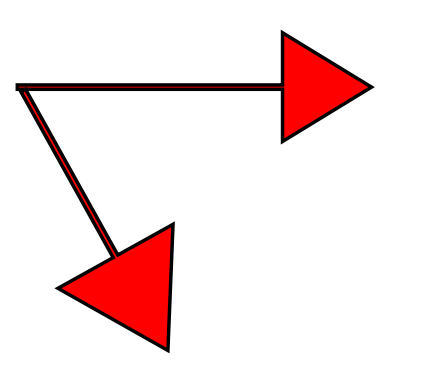

Build molecular
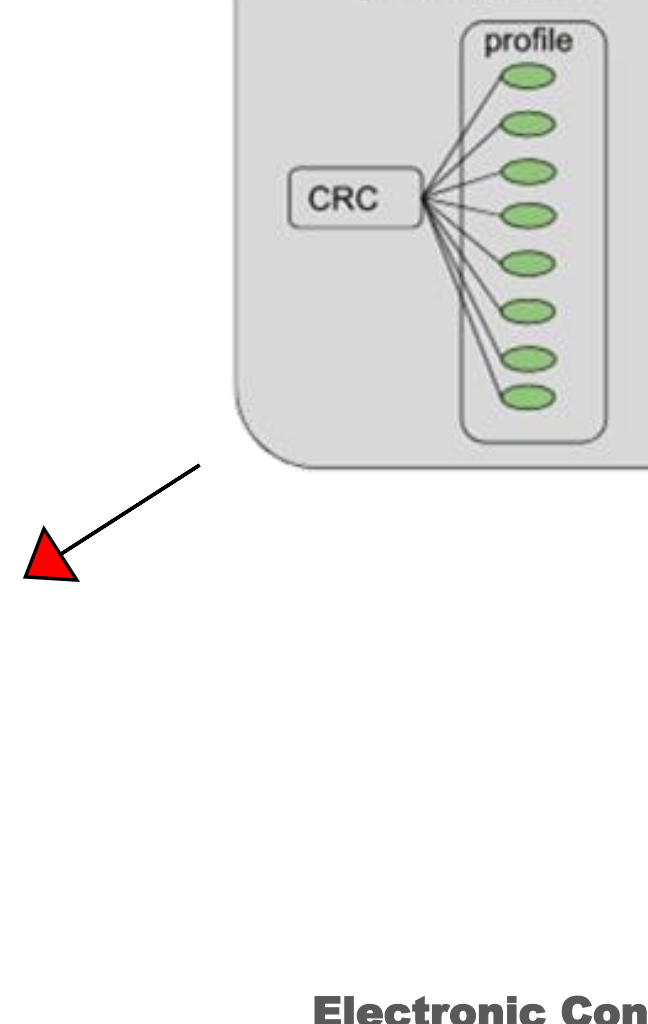
profiles for CRC

Calculate CRC-metabolite profile similarity

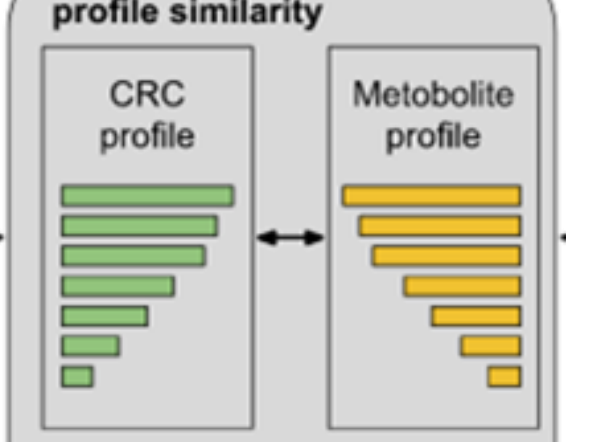

Build molecular profiles for metabolites
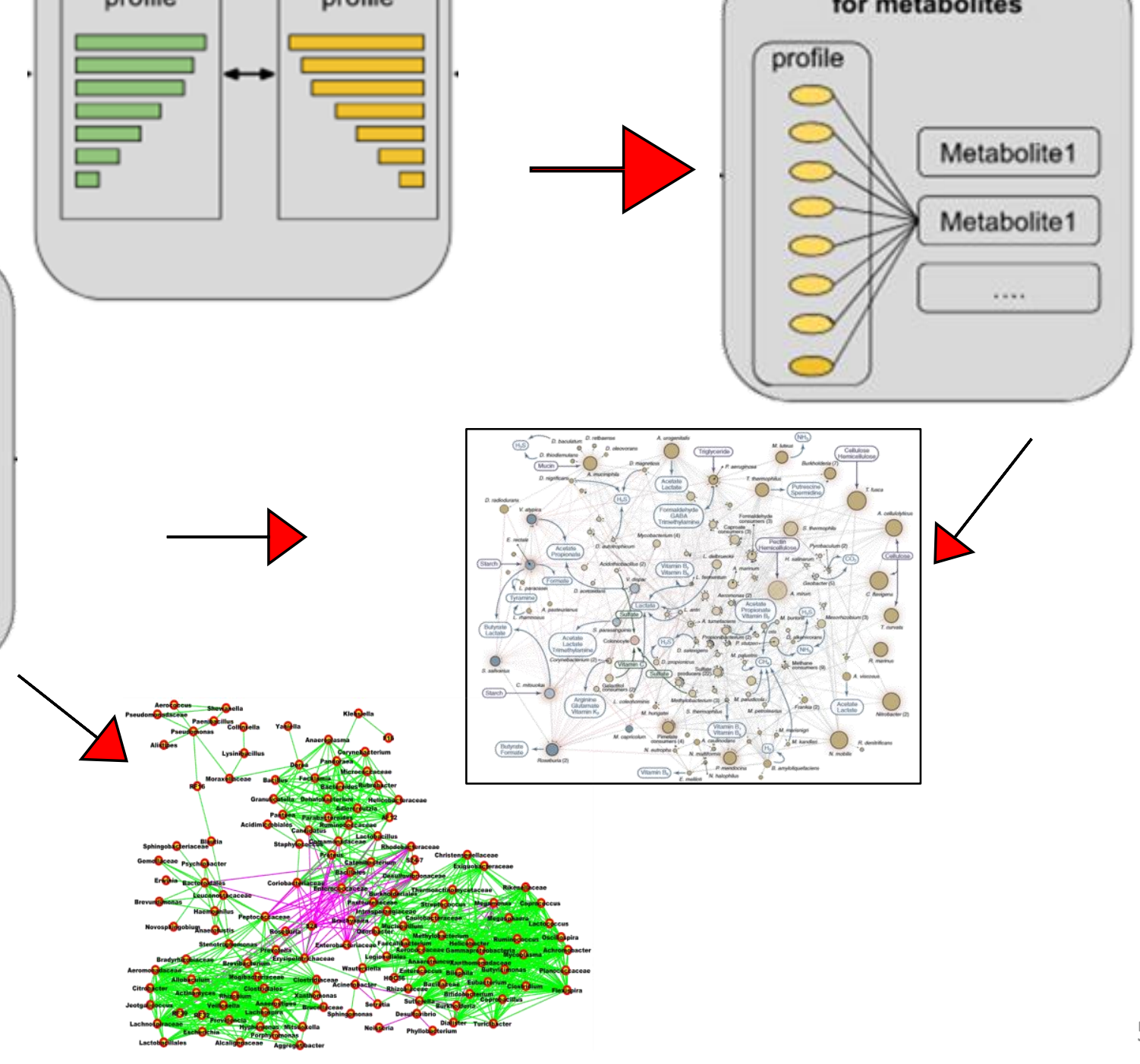

both Alcoholics and CRC patients

A

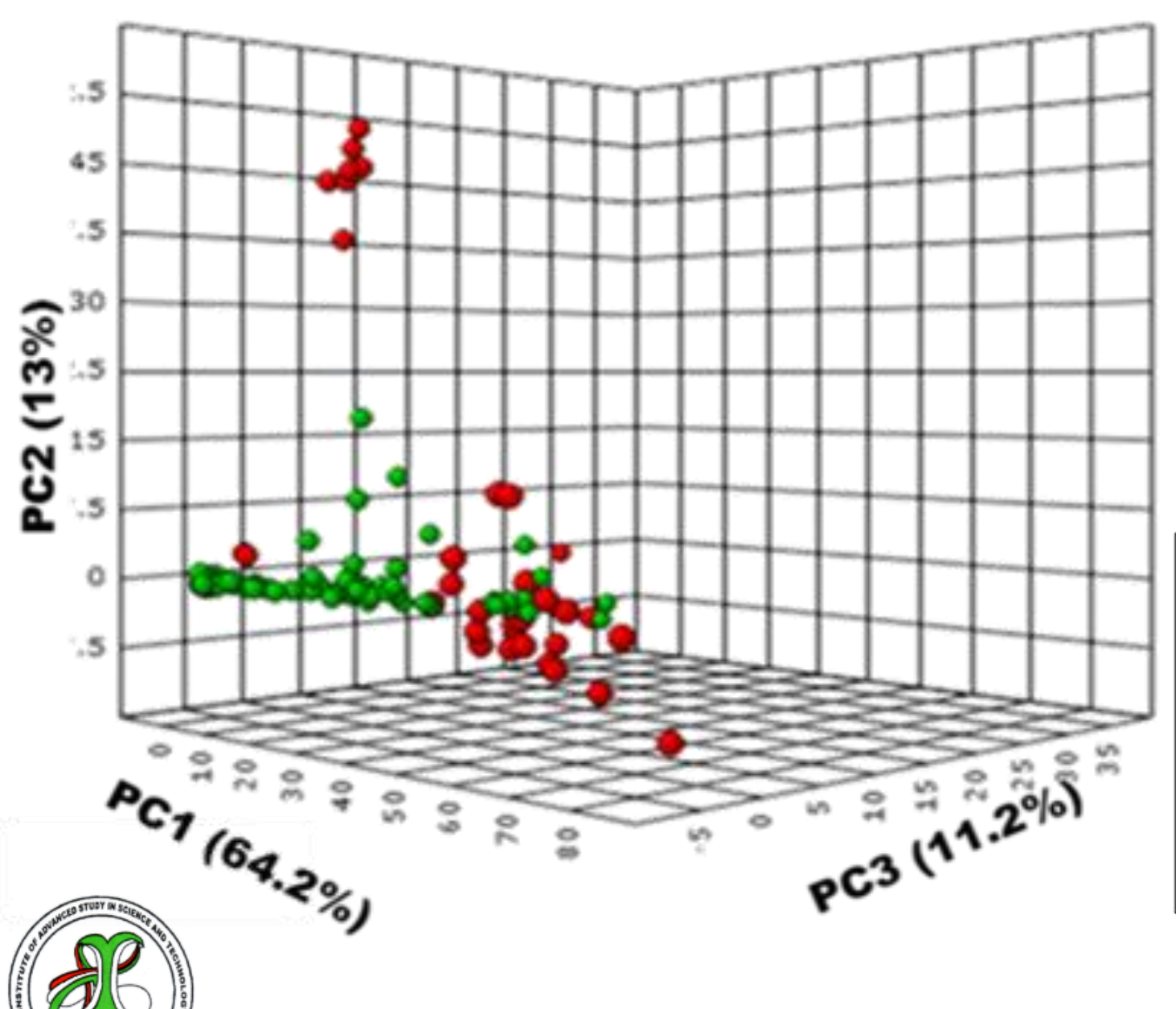

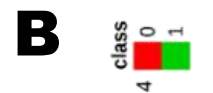
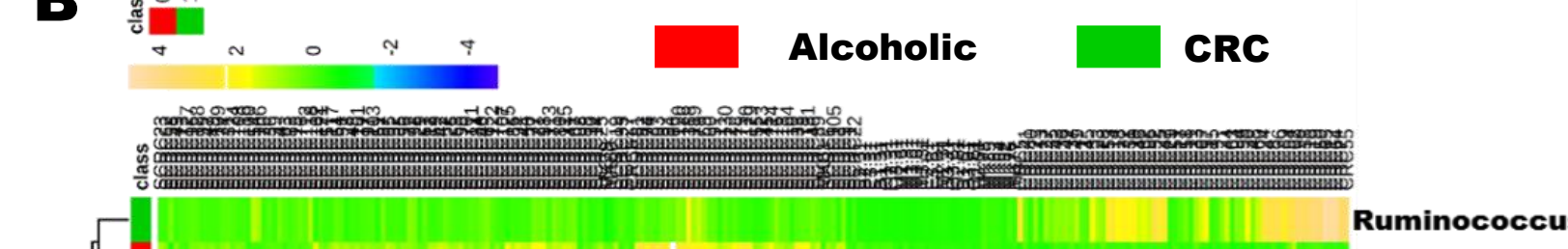

Ruminocos

Ruminococcacea

Eubacterium

Enterobacteriaceae

Bifidobacterium

Fusobacterium

Faecalibacterium

Streptococcus

Lactobacillales

Lactobaciales

Porphyromonadaceae

Bacteroidales

Bacteroides

Actinomyces

Collinsella

Sutterela

Turibacter

Lachnospira

Megamonas

Klebsiella

Helicobacter

Prevotella

Lactobacillus

Lachnospiracha Clostridiales 


\section{Findings ..}
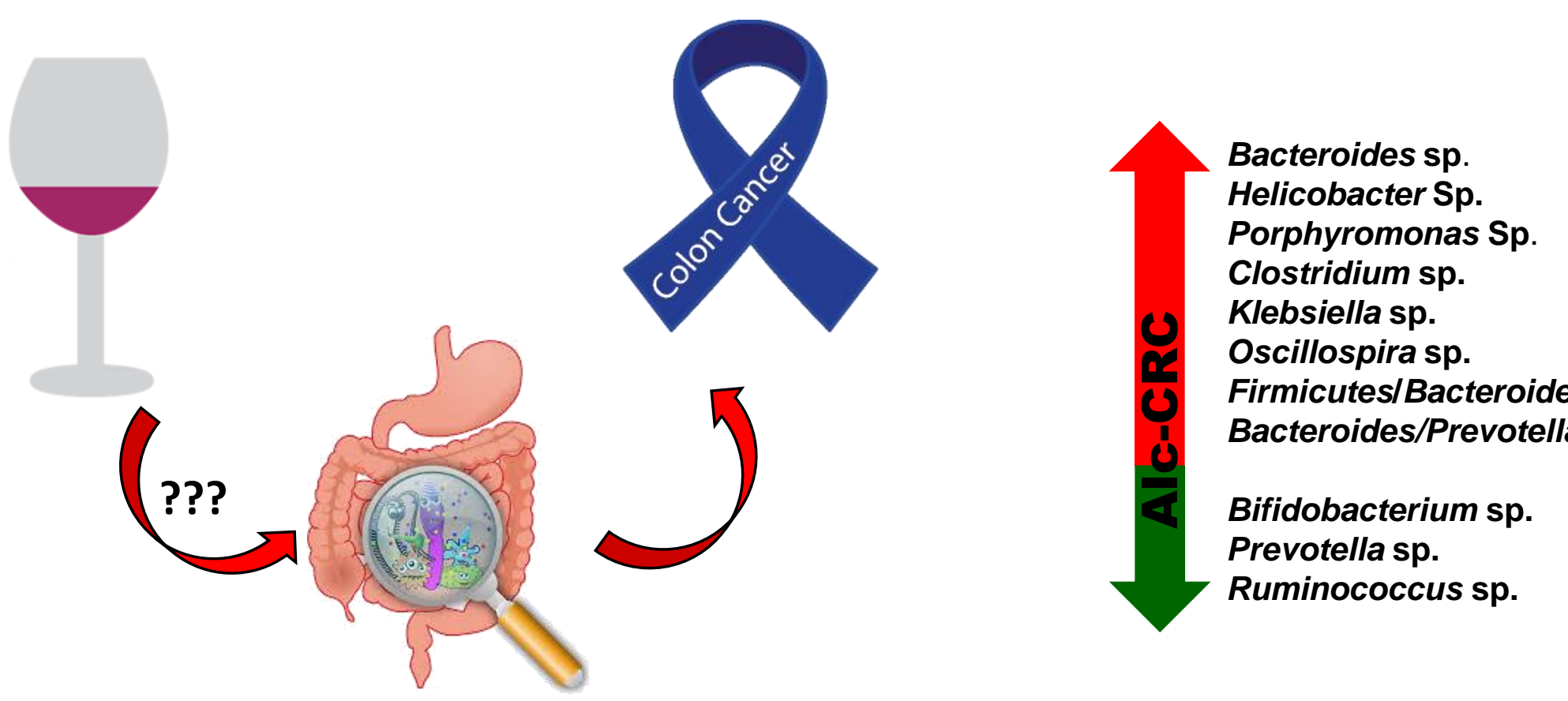


\section{Thank You for listening..}

